\title{
Predict Urinary Tract Infection and to Estimate Causative Bacterial Class in a Philippine Subspecialty Hospital
}

\author{
Verle V Agpaoa', Jefferson B Mendoza1, Arnold Joseph M Fernandez', Januario D Veloso ${ }^{1}$ and Sonu Bhatnagar ${ }^{2}$
}

${ }^{1}$ Department of Laboratory Medicine, National Kidney and Transplant Institute, Philippines

${ }^{2}$ Scientific Affiairs, Sysmex Asia-Pacific Pte Ltd, Singapore

\begin{abstract}
Objective: Urinary tract infection diagnosis by urine culture can be time- and labor- consuming. In the National Kidney and Transplant Institute, up to $75 \%$ of urine culture samples sent to the laboratory yield no growth or insignificant growth. A rapid and reliable screening method to rule out bacterial UTI could reduce culture workload, turnaround time of negative results and also unnecessary antibiotic prescription. Sysmex UF-1000i is urine flow cytometry analyzer which is capable of quantifying urine particles including bacteria and leukocytes. We evaluated the UF-1000i performance for ruling out UTIs and its bacterial scattergram feature to estimate the causative bacterial group, using standard urine culture results as reference method.
\end{abstract}

Methods: 293 urine samples were analyzed using Sysmex UF-1000i analyzer and compared with urine culture results. Bacterial cluster distribution in the bacterial scattergram of positive cultures was also analyzed for causative bacterial class estimation.

Results: Out of 293 urine samples, 104 (35.5\%) samples had bacterial growth of more than $10^{3} \mathrm{CFU} / \mathrm{ml}$ on culture and most common organism isolated was Escherichia coli (28.8\%). The optimum bacterial cut off value of 55/ $\mu$ l and/or leukocytes cut-off value of $27 / \mu \mathrm{l}$ were observed using ROC analysis to rule out UTI. The bacterial scattergram analysis showed $82.7 \%$ concordance with the results of the urine culture, with only $5.8 \%$ discordant results, and $11.5 \%$ had results showing non-specific or wide distribution patterns near the $30^{\circ}$ angle.

Conclusions: Urine samples can be routinely screened for UTI using the Sysmex UF-1000i, and this can improve the overall turnaround time of negative results and reduce laboratory culture workload..

Keywords: Automated urinalysis; Bacterial scattergram; Causative organism; Urinary tract infection; Urine flow cytometer

\section{Introduction}

Urinary tract infection (UTI) is a common medical problem and may range from asymptomatic bacteriuria to severe infection that can lead to serious complications when left untreated. Women are more likely to be affected than men [1]. Specific subpopulations are at increased risk of UTI, including infants and children, pregnant women, diabetic and immunocompromised patients, the elderly and those with underlying urologic abnormalities [1]. Thus, prompt diagnosis is important for proper and timely management.

Urine culture is considered to be the gold standard to diagnose UTI. However, the performance of urine culture is laborious and time consuming, with results usually released after 3 to 4 days. Moreover, studies have proven that up to $80 \%$ of urine samples subjected to urine culture are reported negative for bacteria which make urine culture less cost-effective [2].

Several other methods have been used to evaluate urine for the presence of leukocytes or pyuria to screen for UTI. The dipstick leukocyte esterase test is a rapid and inexpensive test that detects esterase, an enzyme released by white blood cells. However, this test has low sensitivity and a high false-positivity since this can be affected by contamination, often by vaginal secretions [3]. Microscopic examination of urine samples, on the other hand, is used to look for formed cellular elements, casts, bacteria, yeast, parasites and crystals. However, the procedure is time consuming and the results of this method of detection vary with each observer [2].

Sysmex urine flow cytometer UF-1000i (Sysmex Corporation, Kobe, Japan) uses a red semiconductor laser that can quantitatively measure red blood cells (RBC), white blood cells (WBC), epithelial cells, casts and bacteria in non-centrifuged urine in 72 seconds. The instrument has separate sediment and bacteria channels and uses a single polymethine dye for both counting channels. Specific diluents dissolve disturbing salts in the reaction chamber of the sediment channel and allow sensitive staining of urinary formed elements. In the reaction chamber of the bacteria channel, specific diluents also prevent non-specific staining of non-bacterial particles (e.g. red blood cells and casts) by lysing, while the polymethine dye stains bacterial nucleic acids. This provides an accurate quantitative bacterial count without interference of cell debris. The stained particles pass through the center of the fluidic stream one by one at a high velocity. As the stained urine particles cross the laser beam, fluorescent light is emitted and the signals are analyzed according to appropriate particle types. Particle characterization and identification are based on detection of fluorescence (Fl), forward-scatter light (Fsc) and side-scatter light (Ssc) and are graphically supplemented with scattergrams. At the medical laboratory of the National Kidney and Transplant Institute (NKTI), a tertiary subspecialty hospital for renal diseases in the Philippines, urine samples account for majority of the specimens analyzed for routine

${ }^{*}$ Corresponding author: Sonu Bhatnagar, Scientific Affiairs, Sysmex Asia-Pacific Pte Ltd, 9 Tampines Grande, \#06-18, Singapore-520735, Tel: +65 91550275; E-mail: Bhatnagar.sonu@sysmex-ap.com

Received January 21, 2015; Accepted February 19, 2015; Published February 25, 2015

Citation: Agpaoa VV, Mendoza BJ, Fernandezi AJM, Veloso JD, Bhatnagar S (2015) Predict Urinary Tract Infection and to Estimate Causative Bacteria Class in a Philippine Subspecialty Hospital J Nephrol Ther 5: 194. doi: 10.4172/2161-0959.1000194

Copyright: (c) 2015 Agpaoa VV, et al. This is an open-access article distributed under the terms of the Creative Commons Attribution License, which permits unrestricted use, distribution, and reproduction in any medium, provided the original author and source are credited. 
tests, especially among UTI suspected cases. Quantitative evaluation and bacterial identification by urine culture is usually used to diagnose UTI, however, only about $25 \%$ of urine samples yield positive results. An accurate and reliable screening method that readily detects negative urine samples is needed to avoid unnecessary culture tests and to minimize total expenditure on urine sample processing. In order for a true negative urine sample to be correctly classified as such, the screening method should have a high sensitivity and negative predictive value. This can translate in improved detection efficiency and reduction of laboratory costs. The rapid reporting of negative results can also aid the clinicians in prompt and proper management.

Various studies have been reported using UF-1000i as a screening tool for negative UTI results and different cut-off values have been purposed for UTI detection [4-6]. No study has been reported till now to establish optimum cut-off values for the Philippine population.

Thus, the study was designed to determine the utility of Sysmex UF$1000 \mathrm{i}$ in the tertiary sub-specialty hospital as a rapid screening test in predicting negative UTI. The study also aimed to determine optimum cut-off values for leukocytes and bacteria to select urine samples for bacterial cultures, and to evaluate if the distribution of the bacteria cluster on the scattergram can presumptively differentiate bacteria as gram-positive or gram-negative as reported previously $[7,8]$.

\section{Materials and Methods}

From October 2013 to December 2013, we prospectively studied 293 urine samples submitted for urine culture to the Clinical Microbiology Section of the NKTI Medical Laboratory. Both male and female non-transplant out-patients from 18 to 80 years of age were included in the study. Post-transplant patients, patients with urinary diversions, orthotopic neobladder with bowel segments, indwelling urinary catheters, stents and other foreign body devices inserted in the urinary bladder and along the urinary tract, and urine samples obtained via suprapubic bladder aspirate were excluded, as well as patients with current history of antibiotic use. The study was reviewed and approved by the NKTI Technical Review Board and written consent and pertinent clinical data were obtained using an informed consent form (IFC) and questionnaire approved by the NKTI Research Ethics Committee.

Two hundred and ninety-three clean-catch mid-stream urine samples collected in a wide-rimmed preservative-free sterile container were processed within thirty minutes after collection. Both male and female participants were given instructions on proper urine collection. The urine samples were first subjected to urine culture according to the current Microbiology section work instruction manual to avoid contamination by manipulation. After gentle inversion, $1 \mu \mathrm{L}(0.001$ $\mathrm{ml}$ ) of urine was inoculated onto $1 / 3$ of a blood agar plate (BAP) by making a straight line down for primary streak and making a series of uninterrupted streak procedure using a calibrated loop; and another $1 \mu \mathrm{L}$ of the same sample was inoculated to $1 / 3$ of a MacConkey agar plate (MAC) by interrupted streak procedure. Both agar plates were incubated at $35-37^{\circ} \mathrm{C}$ for $18-24$ hours, then colony counting was carried out and expressed as number of colony forming units (CFU)/ $\mathrm{ml}$. The culture plates were interpreted based on the current Clinical and Laboratory Standards Institute (CLSI) guidelines. A culture was considered positive if there is growth of more than $10^{2} \mathrm{CFU} /$ $\mathrm{ml}$ in a symptomatic female, $10^{3} \mathrm{CFU} / \mathrm{ml}$ in a symptomatic male or $10^{5} \mathrm{CFU} / \mathrm{ml}$ in voided urine of an asymptomatic patient. A sample was considered negative for UTI if there was no growth or there was $<10^{3} \mathrm{CFU} / \mathrm{ml}$ bacterial growth, which is considered as insignificant growth. For a positive urine culture, isolated microorganisms were identified using conventional biochemical procedures. Lactobacillus, diphtheroids, Bacillus species, other types of coagulase negative Staphylococci and Streptococcus viridans group isolates were considered to be contaminants [9] and were excluded. Specimens yielding mixed growth of gram-positive and negative bacteria, fungi and mycobacteria were also excluded.

The following bacteria were considered to be uropathogens: Enterobacteriaceae, non-fermentative gram-negative bacilli, Staphylococcus saprophyticus, Enterococcus species, Staphylococcus aureus, Group A, B, C or G Streptococci, Streptococcus pneumoniae, Corynebacterium urealyticum or Aerococcus species (i.e. $>65$ years of age) and yeasts [9].

Within 10 minutes after inoculation of the culture, the urine samples were transferred to the Clinical Microscopy section and were immediately analyzed using the urine particle analyzer Sysmex UF1000i (quantitative) and bacterial and leukocytes counts were obtained. Manual microscopic examination was done to verify abnormal samples flagged by the laboratory's flagging criteria for pathological casts, yeastlike organisms, sperm, etc. The bacterial scattergrams of urine samples with positive urine cultures were printed and their angle of bacterial distribution were classified as having greater than, less than or wide angle distribution in reference to the $30^{\circ}$ angle [8].

\section{Data analysis}

The sensitivity, specificity, positive predictive value (PPV) and negative predictive value (NPV) for different cut-off values for leukocyte and bacterial counts were calculated using the urine culture as the reference. Receiving operator characteristics (ROC) curves for bacteria and leukocytes were plotted to assess the cut-off values using the SPSS 19.0 software. The Area Under the ROC (AUROC) curves were also computed. The number of urine cultures that could have been avoided using the optimum cut-off values was determined. Bacterial scattergram results were analyzed using descriptive statistics.

\section{Results}

There were 189 female and 104 male participants in the study. Among the 293 urine samples, 104 (35.5\%) were positive for bacterial growth of more than $10^{3} \mathrm{CFU} / \mathrm{ml}$ in symptomatic patients and more than $10^{5} \mathrm{CFU} / \mathrm{ml}$ in asymptomatic patients, $130(44.4 \%)$ were negative and $59(20.1 \%)$ were excluded due to growth of contaminants, polymicrobial growth ( $>3$ microorganisms) and fungi. The majority of the positive urine samples $(73 / 104 ; 70.2 \%)$ and excluded samples that yielded contaminants, polymicrobial infection or yeasts $(54 / 59$; $91.5 \%)$ were from female participants. Seventy-seven percent $(80 / 104)$ of the positive cultures yielded gram-negative bacteria and 24 (23\%) grew gram-positive bacteria. Escherichia coli was the most common organism isolated (28.8\%), followed by Staphyloccocus spp. (13.5\%), Klebsiella pneumoniae (9.6\%), Escherichia coli-ESBL producer (7.7\%), Klebsiella pneumonia-ESBL producer (3.8\%), Acinetobacter lwoffii (2.9\%), Enterococcus faecalis (2.9\%), Klebsiella and E. coli co-infection (2.9\%) and Pseudomonas aeruginosa (2.9\%) (Table 1).

The receiving operator characteristic (ROC) curves for leukocytes and bacterial counts are shown in Figure 1. The area under the ROC (AUROC) curve of the UF-1000i for bacterial count (0.918) was higher than the AUROC for the leukocytes count (0.808) when greater than $10^{3} \mathrm{CFU} / \mathrm{ml}$ was considered as the standard for a positive urine culture (Table 2). Tables 3 and 4 lists the summary of the sensitivities, 
Citation: Agpaoa VV, Mendoza BJ, Fernandezi AJM, Veloso JD, Bhatnagar S (2015) Predict Urinary Tract Infection and to Estimate Causative Bacterial Class in a Philippine Subspecialty Hospital J Nephrol Ther 5: 194. doi:10.4172/2161-0959.1000194

Page 3 of 6

\begin{tabular}{|c|c|}
\hline Bacteria & Frequency \\
\hline Escherichia coli & $30(28.8 \%)$ \\
\hline Staphylococcus spp. & $14(13.5 \%)$ \\
\hline Klebsiella pneumonia & $10(9.6 \%)$ \\
\hline Escherichia coli (ESBL) & $8(7.7 \%)$ \\
\hline Klebsiella pneumoniae (ESBL) & $4(3.8 \%)$ \\
\hline Acinetobacter Iwoffii & $3(2.9 \%)$ \\
\hline Enterococcus faecalis & $3(2.9 \%)$ \\
\hline Klebsiella pneumoniae, Escherichia coli & $3(2.9 \%)$ \\
\hline Pseudomonas aeruginosa & $3(2.9 \%)$ \\
\hline Enterobacter aerogenes & $2(1.9 \%)$ \\
\hline Enterococcus spp. & $2(1.9 \%)$ \\
\hline Klebsiella ozanae & $2(1.9 \%)$ \\
\hline Streptococcus agalactiae & $2(1.9 \%)$ \\
\hline Acinetobacter baumanii, Serratia marcescens & $1(0.96 \%)$ \\
\hline Enterobacter agglomerans & $1(0.96 \%)$ \\
\hline Enterobacter agglomerans (AmpC+), Escherichia coli & $1(0.96 \%)$ \\
\hline Escherichia coli (AmpC+) & $1(0.96 \%)$ \\
\hline Escherichia coli, Enterobacter aerogenes (AmpC+) & $1(0.96 \%)$ \\
\hline $\begin{array}{l}\text { Escherichia coli, Proteus mirabilis, Klebsiella } \\
\text { pneumoniae }\end{array}$ & $1(0.96 \%)$ \\
\hline Escherichia vulneri & $1(0.96 \%)$ \\
\hline Klebsiella ozanae (AmpC+) & $1(0.96 \%)$ \\
\hline Klebsiella pneumoniae (AmpC+) & $1(0.96 \%)$ \\
\hline Klebsiella pneumoniae (ESBL), Escherichia coli (ESBL) & $1(0.96 \%)$ \\
\hline Proteus mirabilis, Escherichia coli & $1(0.96 \%)$ \\
\hline Proteus vulgaris & $1(0.96 \%)$ \\
\hline Pseudomonas aeruginosa (AmpC+) & $1(0.96 \%)$ \\
\hline $\begin{array}{l}\text { Pseudomonas aeruginosa }(\mathrm{AmpC}+) \text {, Escherichia coli } \\
\text { (ESBL) }\end{array}$ & $1(0.96 \%)$ \\
\hline Shigella sonnei & $1(0.96 \%)$ \\
\hline Staphylococcus aureus (MRSA) & $1(0.96 \%)$ \\
\hline Staphylococcus aureus, Gamma streptococci & $1(0.96 \%)$ \\
\hline Staphylococcus saprophyticus & $1(0.96 \%)$ \\
\hline Total & 104 \\
\hline
\end{tabular}

Table 1: List of Bacteria found in 104 Urine Cultures

specificities, PPV and NPV of different cut off values for the bacterial and leukocytes counts, respectively.

The optimum cutoff value for the bacterial count was $55 / \mu \mathrm{l}$ on the ROC curve, achieving a sensitivity of $94.2 \%$ and specificity of $72.3 \%$, PPV of $77.3 \%$ and NPV of $92.6 \%$. With this cut off, $39 \%$ of unnecessary cultures could be avoided with a $2.56 \%$ false-negative rate.

The cutoff value for the leukocytes count, on the other hand, was $27 / \mu$ l on the ROC curve with a sensitivity of $84.6 \%$, specificity of $65.4 \%$, PPV of $71 \%$ and NPV of $81 \%$ and allows the elimination of $40.4 \%$ of unnecessary cultures but with a $6.84 \%$ false negative rate.

If we consider the bacterial count and leukocytes count cut-off values together, there was an increase in sensitivity to $95.2 \%$, specificity $82.3 \%$, PPV $81.2 \%$, NPV $95.5 \%$ and the number of unnecessary cultures that could be avoided improved to $43.6 \%$ with only a $2.14 \%$ false negative rate.

The bacterial scattergram of the 104 urine samples with positive cultures revealed that $86(82.7 \%)$ scattergram results were in agreement with the results of the urine culture, while $6(5.8 \%)$ results were discordant. Seven $(6.7 \%)$ cases had a wide distribution pattern or had dots distributed above and below the $30^{\circ}$ reference angle and $5(4.8 \%)$ cases showed scattered, non-specific bacterial distribution. Examples

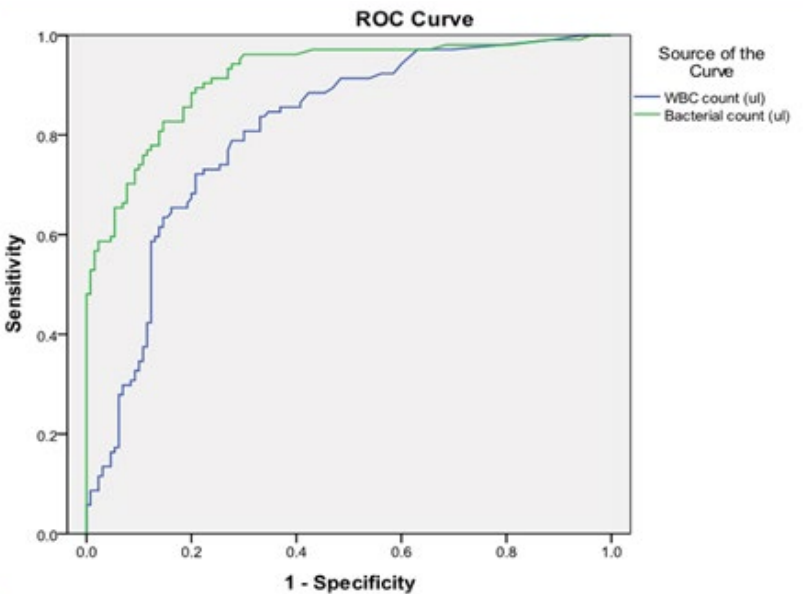

Figure 1: ROC Curve of Bacterial and WBC Count

\begin{tabular}{|c|c|c|c|c|c|}
\hline \multirow{2}{*}{$\begin{array}{c}\text { Test Result } \\
\text { Variable }\end{array}$} & \multirow{2}{*}{ Area } & \multirow{2}{*}{$\begin{array}{c}\text { Standard } \\
\text { Error }\end{array}$} & \multirow{2}{*}{ p-value } & \multicolumn{2}{|c|}{$\begin{array}{c}\text { Asymptotic } 95 \% \text { Confidence } \\
\text { Interval }\end{array}$} \\
\hline & & & & $\begin{array}{l}\text { Lower } \\
\text { Bound }\end{array}$ & Upper Bound \\
\hline WBC $(\mu \mathrm{l})$ & 0.808 & 0.029 & 0 & 0.752 & 0.864 \\
\hline BAC $(\mu l)$ & 0.918 & 0.018 & 0 & 0.882 & 0.954 \\
\hline
\end{tabular}

Table 2: Area Under the Curve (AUC) for Bacteria (BAC) and White Blood Cell (WBC) Counts

of the different bacterial distribution patterns are shown in Figure 2. Among the concordant results, 69 (66.3\%) samples had bacterial distribution of less than $30^{\circ}$ on the scattergram and grew gram-negative bacteria on culture and 17 (16.3\%) samples had a bacterial distribution of greater than $30^{\circ}$ and yielded gram positive bacteria on culture. Of the 6 discordant results, 4 samples showed bacterial distribution with an angle less than $30^{\circ}$ but yielded Staphylococci spp, with one case yielding $S$. aureus (MRSA), while 2 samples both had bacterial distribution of more than $30^{\circ}$ but grew gram-negative bacteria, one grew E. coli and the other yielded a co-infection of Klebsiella pneumoniae (ESBL) and E. coli (ESBL) (Table 5).

\section{Discussion}

Urinary tract infection is a common disease that requires prompt diagnosis and treatment. Unfortunately, urine culture, the gold standard for the diagnosis of UTI, is time-consuming, expensive and often yields negative results. This not only affects physicians' decision making and patients' treatment, but laboratory workload and management as well. The new generation fully automated Sysmex UF-1000i urine analyzer allows a more accurate analysis of RBC, leukocytes, bacteria, epithelial cells and casts in urine in a very short time, and can thus contribute to the screening of UTI [7] in contrast to other urine screening methods such as the dipstick leukocyte esterase test and urinary sediment microscopy which show varied and inconsistent results [2]. In this study, we explored the utility of the automated urine particle analyzer Sysmex UF-1000i as a rapid screening tool for UTI and investigated the ability of its bacterial scattergram to predict bacterial class in order to help physicians in choosing the appropriate empiric treatment.

The study had more female subjects diagnosed with UTI compared to males [1]. This can be attributed to the short urethra in females and its proximity to the external female genitalia and anus. Furthermore, the women in this study are within the reproductive age group and 
Citation: Agpaoa VV, Mendoza BJ, Fernandezi AJM, Veloso JD, Bhatnagar S (2015) Predict Urinary Tract Infection and to Estimate Causative Bacterial Class in a Philippine Subspecialty Hospital J Nephrol Ther 5: 194. doi:10.4172/2161-0959.1000194

Page 4 of 6

\begin{tabular}{|c|c|c|c|c|c|c|c|}
\hline $\begin{array}{c}\text { Bacterial Cut- } \\
\text { off }(\boldsymbol{\mu l})\end{array}$ & $\begin{array}{c}\text { Sensitivity or True } \\
\text { Positive(TP) }\end{array}$ & $\begin{array}{c}\text { Specificity or True } \\
\text { Negative(TN) }\end{array}$ & $\begin{array}{c}\text { False Negative(FN) } \\
\text { =1-Sensitivity }\end{array}$ & $\begin{array}{c}\text { False Positive(FP) } \\
\text { =1-Specificity }\end{array}$ & PPV & NPV & Reduction of Cultures \\
\hline-1.0 & 1.000 & 0.000 & 0.000 & 1.000 & 0.500 & 1.000 & 0.000 \\
\hline 10.5 & 0.971 & 0.462 & 0.029 & 0.538 & 0.643 & 0.941 & 0.245 \\
\hline 25.0 & 0.962 & 0.677 & 0.038 & 0.323 & 0.749 & 0.946 & 0.358 \\
\hline 55.0 & 0.942 & 0.723 & 0.058 & 0.277 & 0.773 & 0.926 & 0.390 \\
\hline 85.0 & 0.913 & 0.762 & 0.087 & 0.238 & 0.793 & 0.898 & 0.424 \\
\hline 103.0 & 0.885 & 0.792 & 0.115 & 0.208 & 0.810 & 0.873 & 0.454 \\
\hline 213.0 & 0.808 & 0.854 & 0.194 & 0.146 & 0.847 & 0.816 & 0.523 \\
\hline 301.5 & 0.769 & 0.885 & 0.231 & 0.115 & 0.870 & 0.793 & 0.558 \\
\hline 400.0 & 0.702 & 0.923 & 0.298 & 0.077 & 0.901 & 0.756 & \\
\hline 528.0 & 0.654 & 0.931 & 0.346 & 0.069 & 0.904 & 0.729 & 0.611 \\
\hline 737.0 & 0.625 & 0.946 & 0.375 & 0.054 & 0.921 & 0.716 & 0.638 \\
\hline 1019.0 & 0.587 & 0.977 & 0.413 & 0.023 & 0.962 & 0.703 & 0.661 \\
\hline 1461.0 & 0.519 & 0.992 & 0.481 & 0.008 & 0.985 & 0.674 & 0.695 \\
\hline 2139.0 & 0.481 & 1.000 & 0.519 & 0.000 & 1.000 & 0.658 & 0.737 \\
\hline
\end{tabular}

Table 3: Summary of Sensitivity, Specificity, Negative Predictive Value, Positive Predictive Value and Reduction of Cultures of UF-1000i according to bacterial count cut-off values in 234 specimens

\begin{tabular}{|c|c|c|c|c|c|c|c|}
\hline $\begin{array}{c}\text { WBC Cut-off } \\
(\mu \mathrm{l})\end{array}$ & $\begin{array}{c}\text { Sensitivity or True } \\
\text { Positive(TP) }\end{array}$ & $\begin{array}{l}\text { Specificity or True } \\
\text { Negative(TN) }\end{array}$ & $\begin{array}{c}\text { False Negative(FN) = } \\
\text { 1-Sensitivity }\end{array}$ & $\begin{array}{l}\text { False Positive(FP) } \\
=1 \text {-Specificity }\end{array}$ & PPV & NPV & Reduction of Cultures \\
\hline-1 & 1 & 0 & 0 & 1 & 0.5 & 1 & 0 \\
\hline 10.5 & 0.913 & 0.5 & 0.087 & 0.5 & 0.646 & 0.852 & 0.293 \\
\hline 22.5 & 0.856 & 0.615 & 0.144 & 0.385 & 0.69 & 0.81 & 0.38 \\
\hline 27 & 0.846 & 0.654 & 0.154 & 0.346 & 0.71 & 0.81 & 0.404 \\
\hline 50.5 & 0.76 & 0.731 & 0.24 & 0.269 & 0.738 & 0.752 & 0.486 \\
\hline 105 & 0.654 & 0.823 & 0.346 & 0.177 & 0.787 & 0.704 & 0.585 \\
\hline 200 & 0.596 & 0.869 & 0.404 & 0.131 & 0.82 & 0.683 & 0.637 \\
\hline 322.5 & 0.481 & 0.877 & 0.519 & 0.123 & 0.796 & 0.628 & 0.698 \\
\hline 422 & 0.423 & 0.885 & 0.577 & 0.115 & 0.786 & 0.605 & 0.731 \\
\hline 500 & 0.375 & 0.892 & 0.625 & 0.108 & 0.777 & 0.588 & 0.759 \\
\hline 712.5 & 0.327 & 0.908 & 0.673 & 0.092 & 0.78 & 0.574 & 0.79 \\
\hline 1012 & 0.298 & 0.931 & 0.702 & 0.069 & 0.812 & 0.57 & 0.816 \\
\hline 1581 & 0.173 & 0.938 & 0.827 & 0.062 & 0.738 & 0.532 & 0.883 \\
\hline 2013.5 & 0.144 & 0.954 & 0.856 & 0.046 & 0.758 & 0.527 & 0.905 \\
\hline
\end{tabular}

Table 4: Summary of Sensitivity, Specificity, Negative Predictive Value, Positive Predictive Value and Reduction of Cultures of UF-1000i according to WBC count cut-off values in 234 specimens

Bacterial distribution on bacterial scattergram

\begin{tabular}{|c|}
\hline Greater than $30^{\circ}$ angle \\
\hline Less than $30^{\circ}$ angle \\
\hline Wide distribution pattern \\
\hline Non-specific distribution pattern \\
\hline TOTAL
\end{tabular}

Positive Urine Culture $(n=104)$

Gram Positive Bacterial Growth

Gram Negative Bacterial Growth

\begin{tabular}{|c|c|}
\hline 17 & Gram Negative Bacterial Growth \\
\hline 4 & 2 \\
\hline 2 & 69 \\
\hline 3 & 5 \\
\hline 26 & 2 \\
\hline
\end{tabular}

Table 5: Summary of Sensitivity, Specificity, Negative Predictive Value, Positive Predictive Value and Reduction of Cultures of UF-1000i according to WBC count cut-off values in 234 specimens

may be sexually active or pregnant, making them more susceptible to UTI [1].

Setting a low bacterial colony growth $\left(>10^{3} \mathrm{CFU} / \mathrm{ml}\right)$ for the diagnosis of UTI is important in our hospital setting to avoid exclusion of critical patients (i.e. pregnant, immunocompromised, recurrent UTI patients) for whom a low colony count is considered significant [4]. The cut-off values set for bacteria and leukocytes counts of $55 / \mu \mathrm{l}$ and $27 / \mu \mathrm{l}$, respectively, were set as such, taking in consideration the prerequisite of a good screening test which has to have a high sensitivity and negative predictive value, without compromising specificity. The total number of cultures that could be eliminated using these cut-off values was also considered, such that false-negative rates obtained were acceptable for our laboratory. The high sensitivity and negative predictive values of bacterial counts of the UF-1000i in this study are comparable with values obtained by other laboratories around the world [5-7,10, 11]. However, the specificity obtained was lower compared to the studies of Wang et al. and Broeren et al. who set higher bacterial cut-off values, $>100 / \mu \mathrm{l}$ and $230 / \mu \mathrm{l}$, respectively $[5,6]$.

On the ROC analysis, the UF-1000i showed better AUROC for bacteria than leukocytes. This can be due to the efficiency of the dedicated bacteria channel of the analyzer that allowed the exclusion of other cell fragments, debris and mucus from the urine particles. 

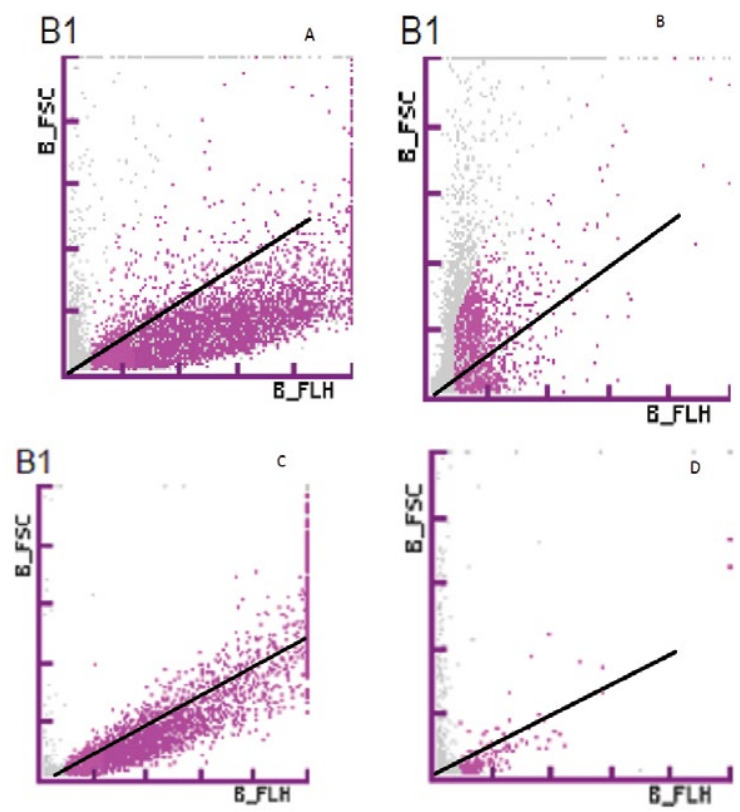

Figure 2: Examples of Different Bacterial Distribution Patterns in the Bacterial Scatter gram: A. Less than $30^{\circ}$ angle; B. More than $30^{\circ}$ angle; C. Wide distribution pattern; D. Non-specific distribution pattern

- B_FSC: Forward scattered light intensity (size)

- B_FLH: Fluorescent light intensity (depth of staining)

The study had a lower sensitivity, specificity, PPV and NPV values obtained from the set leukocytes cut-off value compared to that of the bacterial count. According to the study by Foxman in 2003, the presence of leukocytes in the urine may not be a reliable indicator of UTI alone as this could be due to factors, such as presence of female genital tract infections and persistence of leukocytes after antibiotic treatment of UTI [1], and this could explain why some patients had a high leukocytes count but the culture results was negative. However, by collectively using the bacteria and leukocyte cut-off values, the specificity and PPV of the instrument significantly improved and more unnecessary urine cultures (43\%) can be eliminated with less than $3 \%$ false negative rate, which is acceptable for our hospital's out-patient non-transplant population.

We observed that most of the gram-positive bacteria tend to be distributed above $30^{\circ}$ and that gram-negative bacterial distribution is noted below $30^{\circ}$ in the bacteria scattergram, further supporting the aptness of setting the reference angle at $30^{\circ}$ [8]. There were 4 samples noted with bacterial distribution below the $30^{\circ}$ angle but showed Staphylococci growth including 1 case of $S$. Aureus (MRSA) growth, while the other 2 samples with bacterial distribution above the 30 ' angle showed growth of $E$. coli and a co-infection of two gram-negative ESBL producing organisms. Also, the 7 samples that showed a wideangle distribution pattern near the $30^{\circ}$ angle yielded Enterococcus faecalis, Staphylococcus saprophyticus, Streptococcus agalactiae, Shigella sonnei, Acinetobacter lwoffi, Escherichia vulneri and AmpC+ Klebsiella ozonae. The discordance in results and the wide distribution pattern in these cases may be caused by varied reasons as observed in different studies $[8,12]$. Several studies have shown that Enterococcus $s p p$. may have bacterial distribution patterns similar to gram-negative bacteria due to their formation of short chains [12]. Previous antibiotic treatment and probable development of resistance are also seen to affect bacterial distribution due to filament formation in gramnegative bacilli [8]. Furthermore, the causative bacteria mentioned are commonly observed among complicated and recurrent UTI patients. It is therefore suggested that in patients whose bacterial scattergram show a wide distribution pattern, the presence of complicated UTI should be considered and urine culture is warranted. Further investigations on the factors causing the wide distribution pattern is also suggested. Meanwhile, five samples with a non-specific bacterial distribution had low colony counts $\left(10^{3}-10^{4} \mathrm{CFU} / \mathrm{ml}\right)$ on culture and low bacteria counts (less than $55 / \mu \mathrm{l}$ ) determined by the UF-1000i that resulted in insufficient number of dots to be analyzed.

Not all laboratories offer bacterial culture in Philippines, and if they do, the results of the urine culture are usually available only after 2-5 days from the time of collection. In the majority of cases, the physician at the out-patient department already prescribes empiric antibiotics in patients with signs and symptoms of UTI even before culture results are seen. The utility of the fully automated UF-1000i in rapidly screening urine samples and predicting positive urine culture outcomes with results available within the same day of collection allows the physician to obtain reproducible and reliable results which can help avoid costly and unnecessary laboratory tests and the prescription of unnecessary antibiotics. Moreover, the laboratory is able to reduce culture workloads and laboratory costs by concentrating manual efforts in true positive samples, thus improving workflow and efficiency.

\section{Conclusion}

Analysis of bacterial counts and/or leukocytes counts generated by the UF-1000i may be a useful screening method to exclude UTI and reduce laboratory workload and unnecessary urine cultures. In our setting, we suggest the use of the bacterial count of $55 / \mu \mathrm{l}$ and/or leukocytes count of $27 / \mu$ in order to eliminate $43.6 \%$ of unnecessary 
Citation: Agpaoa VV, Mendoza BJ, Fernandezi AJM, Veloso JD, Bhatnagar S (2015) Predict Urinary Tract Infection and to Estimate Causative Bacterial Class in a Philippine Subspecialty Hospital J Nephrol Ther 5: 194. doi:10.4172/2161-0959.1000194

cultures. Also, the use of bacterial scattergram allows a rapid and accurate estimation of the causative organism that can be a helpful guide in the selection of empiric antimicrobial treatment for UTI.

\section{Acknowledgement}

We would like to thank Elizabeth Garcia, RMT and Bernadette Hapitana, RMT of NKTI for their administrative support and technical expertise; the NKTI Laboratory AP-CP Division staff from the sections of Clinical Microscopy, Microbiology and Laboratory Reception Hall for their support and assistance in the research process; Ms. Zykhar Batulan for the technical support; the NKTI Clinical Trial and Research Unit Technical Review Board and Ethics Research Committee for their valuable suggestions on the earlier drafts of the manuscript; and Dr. Sonu Bhatnagar for her constant support, guidance and generosity in the final editing of this manuscript. This research was supported, in part, by Sysmex Asia Pacific Pte Ltd through a cooperative agreement.

\section{References}

1. Foxman B (2002) Epidemiology of urinary tract infections: incidence, morbidity and economic costs. Am J Med 113: 5S-13S.

2. Kim SY, Kim YJ, Lee SM, Hwang SH, Kim HH, et al. (2007) Evaluation of the Sysmex UF-100 urine cell analyzer as a screening test to reduce the need for urine cultures for community-acquired urinary tract infection. Am J Clin Patho 128: 922-925.

3. Burd EM, Kehl KS (2011) A critical appraisal of the role of the clinical microbiology laboratory in the diagnosis of urinary tract infections. J Clin Microbiol 49: S34-S38.

4. Pieretti B, Brunati P, Pini B, Colzani C, Congedo P, et al.(2010) Diagnosis of bacteriuria and leukocyturia by automated flow cytometry compared with urine culture. J Clin Microbiol 48: 3990-3996.
5. Wang J, Zhang Y, Xu D, Shao W , Lu Y (2010) Evaluation of the Sysmex UF$1000 \mathrm{i}$ for the diagnosis of urinary tract infection. Am J Clin Pathol 133: 577-582.

6. Broeren MAC, Bahçeci S, Vader HL, Arents NL (2011) Screening for urinary tract infection with the Sysmex UF-1000i urine flow cytometer. J Clin Microbiol 49: 1025-1029.

7. Ozawa Hideo, Yajima Naoko and Hideyuki Kobayashi. (2012) Estimation of the causative bacterial group from bacterial scattergrams of the fully automated urine particle analyzer UF-1000i. Sysmex Journal International 22.

8. Muratani T, Kobayashi T, Minamoto $Y$, Ikuno $Y$ and S Migata. (2013) The possibility of the bacterial class estimate using urine from patients with the urinary tract infection by the fully automated urine particle analyzer UF-1000i. Sysmex Journal International 23.

9. Semeniuk H, Noonan J, Gill H, Church D (2002) Evaluation of the coral UTI screen system for rapid automated screening of significant bacteriuria in a regional centralized laboratory. Diagn Microbiol Infect Dis 44: 7-10.

10. Gutierrez-Fernandez J, Lara A, Bautista MF, de Dios Luna J, Polo P, et al.(2012) Performance of the Sysmex UF-1000i in screening for significant bacteriuria before quantitative culture of aerobic/facultative fast-growing bacteria in a reference hospital. Journal of Applied Microbiology 113: 609-614.

11. Jolkkonen S, Paattiniemi E, Karpanoja P, Sarkkinen H (2010) Screening of urine samples by flow cytometry reduces the need for culture. J Clin Microbiol 48: 3117-3121.

12. Yasutake $Y$, Higuchi M, Oda S, Tamura Y, Shimadu T, et al.(2013) Comparisons of the Bact scattergram pattern by fully automated integrated urine analyzer UX-2000 and microscopic examination results using gram stain. Sysmex Journal International 23: 1-10. 